Windows on the Instability Strip 


\title{
The Masses and Luminosities of Globular Cluster RRc Stars
}

\author{
Norman R. Simon ${ }^{1}$ and Christine M. Clement ${ }^{2}$ \\ ${ }^{1}$ Department of Physics and Astronomy, University of Nebraska - Lincoln \\ ${ }^{2}$ Department of Astronomy, University of Toronto
}

\begin{abstract}
A large number of hydrodynamic pulsation models are converged in the first overtone, and their mean properties compared with observations of RRc stars in six globular clusters. The two observed quantities, period and Fourier parameter $\phi_{31}$, lead, via the models, to inferred values for mean mass, luminosity and temperature of the RRc sample in each cluster. We find a narrow range in intracluster RRc luminosity and temperature, but a wider range in mass. At the same time, the intercluster spread is wide in all three parameters. A full discussion of our techniques and results will be given elsewhere (Simon and Clement, in preparation).
\end{abstract}

\section{Introduction}

We begin with the technique of Fourier decomposition (e.g., Simon 1988), in which a Fourier series,

$$
\operatorname{mag}=A_{0}+\Sigma_{j=1}^{n} A_{j} \cos \left(j \omega t+\phi_{j}\right)
$$

is fitted to the observed magnitudes of a pulsating star, and the shape of the light curve quantified in terms of the low-order coefficients, viz., $R_{j 1}=A_{j} / A_{1}, \phi_{j 1}=$ $\phi_{j}-j \phi_{1},(j=1,2,3,4)$. A recent study by Clement, Jankulak and Simon (1992; hereafter CJS) applied this technique to globular cluster RRc stars, focusing on the phase parameter $\phi_{31}$. Figure 1, borrowed from CJS, summarises the results of this study in the form of a plot of $\phi_{31}$ vs. $\log$ (period). Here each symbol represents an RRc star and the differing symbols denote different clusters as indicated in the figure caption, with the cluster's $[\mathrm{Fe} / \mathrm{H}]$ value given in parentheses.

Despite considerable scatter, the data in Figure 1 show two clearly discernable trends: 1) there is an increase of $\phi_{31}$ with period within each cluster; and 2) the clusters are segregated according to metallicity, with the metal-rich clusters lying higher and the metal-poor clusters lower. In what follows we shall use linear and hydrodynamic pulsation models to interpret these data, deriving mean masses and luminosities for the various clusters and reproducing the intracluster relation between $\phi_{31}$ and period. We shall see that a considerable range of masses is indicated, both in a given cluster and among the clusters. 


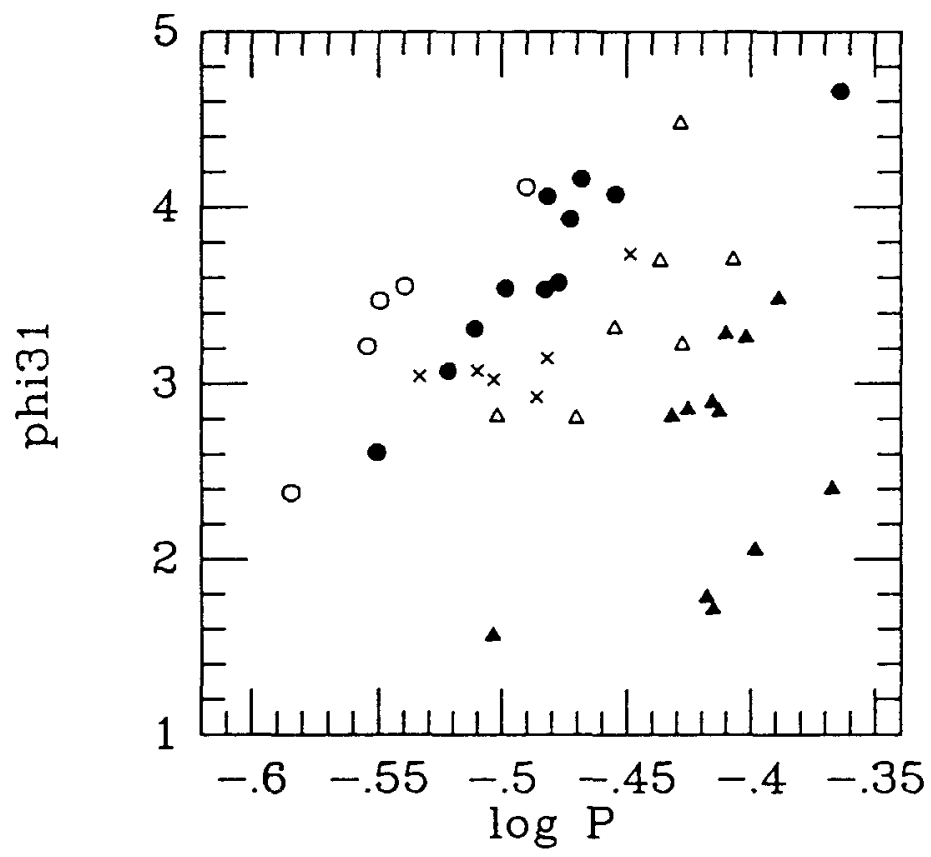

Figure 1. $\phi_{31}$ vs. $\log$ (period) for five globular clusters: open circles - NGC $6171(-0.99)$; filled circles - M5 (-1.40); crosses - M3 (-1.66); open triangles - M53 (-2.04); closed triangles - M15 (-2.17). Numbers in parentheses indicate published values of $[\mathrm{Fe} / \mathrm{H}]$.

\section{The models}

Hydrodynamic pulsation models were perturbed in the first overtone and integrated from 100 to 300 periods to allow the light curves to settle down to their limiting form. An eighth-order Fourier fit was then performed according to Eq. (1), and the value of $\phi_{31}$ extracted for each model. The calculations are very similar to those described by Simon (1990a; hereafter S90a) except that OPAL opacities (Rogers and Iglesias 1992) were employed. The chemical composition, dictated by then available opacity tables, was $X=0.7$, and $Z=0.001$ or 0.0001 (Anders-Grevesse mixture).

Table 1 presents the results of these calculations, giving for each mass (solar units), the values of $\log L$ (solar units), $T_{\text {eff }}$, metal abundance (see code beneath table), pulsation period (days), and Fourier parameters $R_{21}, \phi_{21}, R_{31}$ and $\phi_{31}$. The structure of the present models in general, and the $\phi_{31}$ values in particular, is very similar to that found by $\mathrm{S} 90 \mathrm{a}$ using Los Alamos opacities. The opacity law does not play a crucial role in determining $\phi_{31}$. Neither does the metallicity. Figure 2 shows a plot of $\phi_{31}(Z=0.001)$ vs. $\phi_{31}(Z=0.0001)$ for pairs of models with the same values of $M, \log L$ and $T_{\text {eff. }}$ A linear fit to these points yields a slope 1.000 and zero point 0.019 . It seems likely from Figure 1 that the differences in $\phi_{31}$ are random, probably caused by slightly different convergence properties in the models, i.e., $\phi_{31}$ 

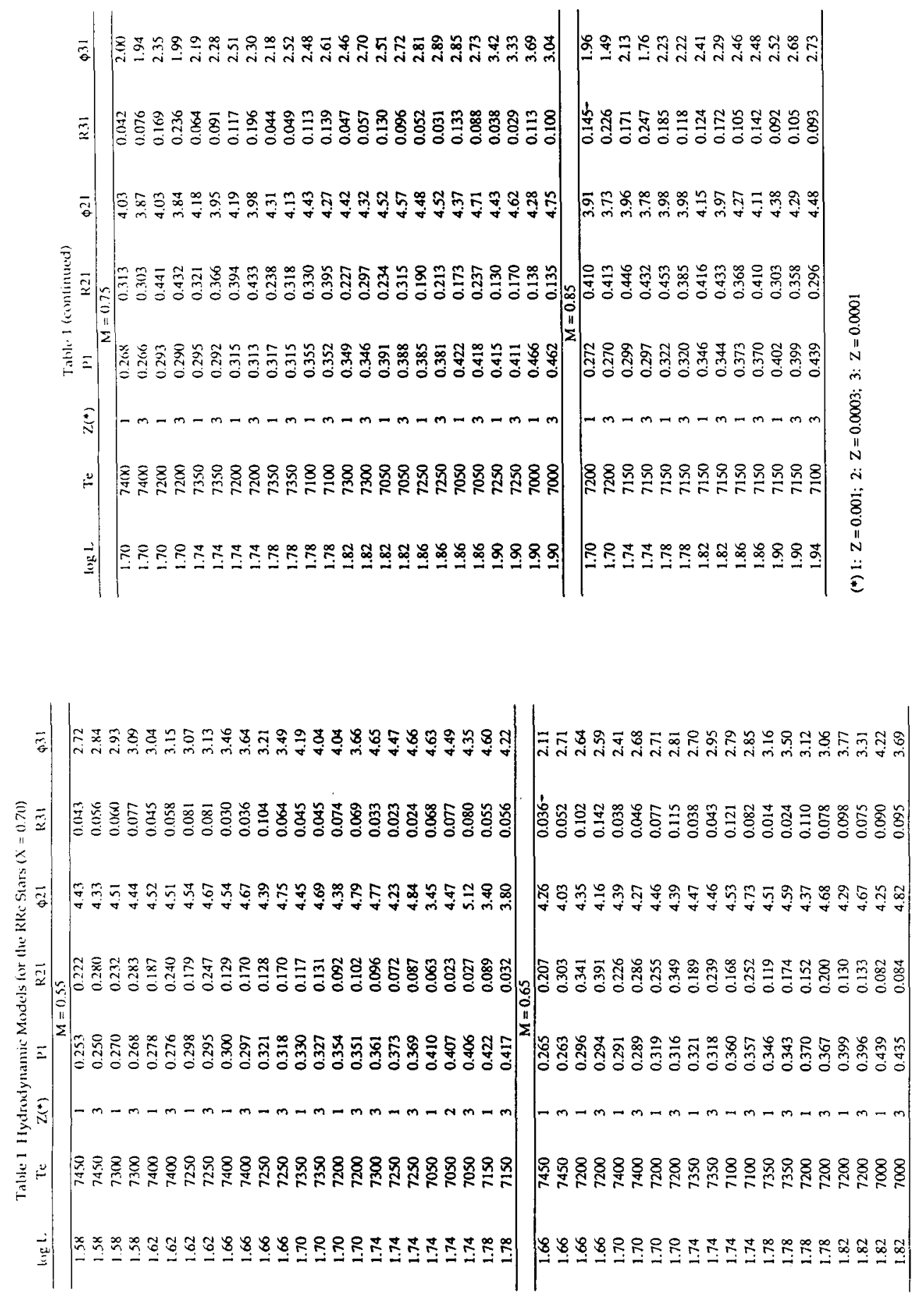
approaching its limiting value by a slightly different path. If this is the case, then the mean difference between the $\phi_{31}$ pairs (which turns out to be $\left|\delta \phi_{31}\right|=0.2$ ) may be taken as a measure of the uncertainty in determining $\phi_{31}$ for a given model: namely, $\Delta \phi_{31}=10.1$. This number is in accord with error estimates made in a different way by $\mathrm{S} 90 \mathrm{a}$.

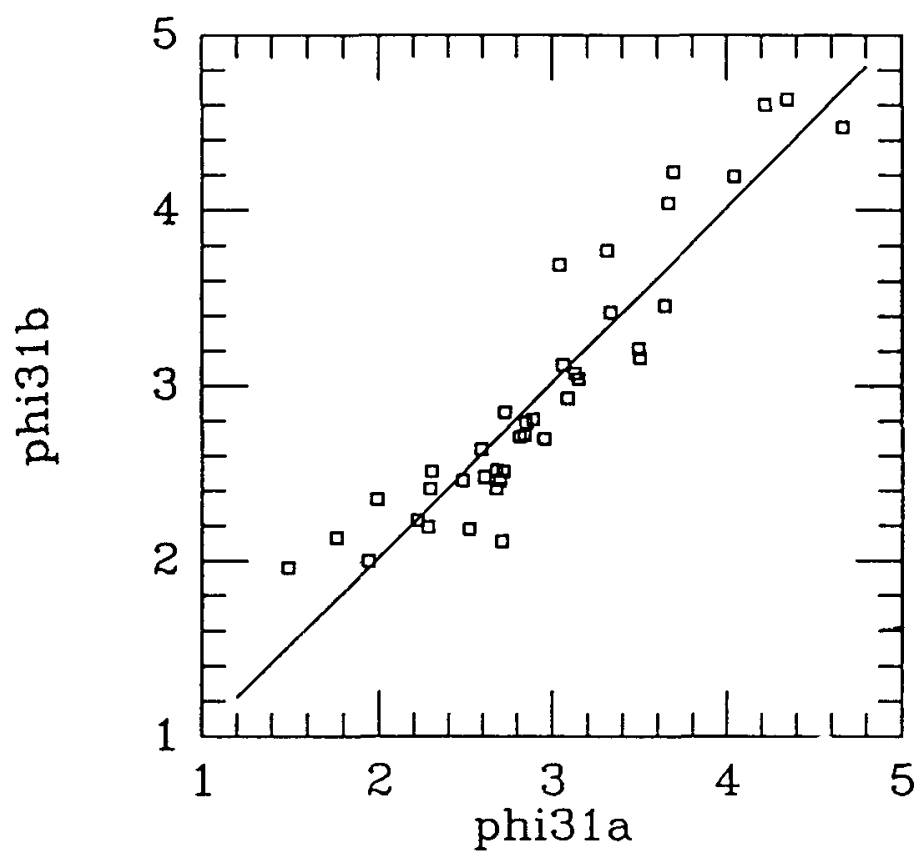

Figure 2. $\phi_{31}$ values for pairs of hydrodynamic models with the same $M, L$ and $T_{\text {eff }}$ but two different values of the metallicity: "phi31a" corresponds to $Z=0.0001$; "phi31b" to $Z=0.001$. The solid line is a least-squares fit to the points.

The model grid in Table 1 was chosen in a similar manner to that in S90a. For each mass and luminosity, models were calculated near the first-overtone blue edge and near the fundamental-mode blue edge, thus spanning the region where first-overtone pulsation is expected. An examination of Table 1 discloses trends in the models which are reminiscent of those in the observations: 1) for given mass there is an increase of $\phi_{31}$ with period; and 2) the higher the mass, the lower the values of $\phi_{31}$.

Finally, we point out the crucial feature that emerges from both the present calculations and those of S90 - over 120 hydrodynamic models, in all: The phase parameter $\phi_{31}$ is determined essentially by mass and luminosity and is hardly sensitive to metallicity, helium abundance, effective temperature and the choice of opacity law (see also Simon 1989; Simon 1990b). It is this property that shall allow us to use $\phi_{31}$ to determine masses and luminosities for the RRc stars.

\section{Theory vs. observations}

In order to make the comparison with observations, we shall describe the average 
properties of the calculations by means of parameter fits to the model sample. Although the models of Table 1 were calculated for a single value of the helium parameter, $Y \simeq 0.30$, the properties of $\phi_{31}$ described just above make it possible to use linear calculations to generalise the models to other values of $Y$. This has been done, resulting in a grid of 140 models, each characterized by six parameters: $M, \log L, Y$, $T_{\text {eff }}, \phi_{31}$ and the first-overtone period, $P_{1}$. Parameter fits to these models then yield relations of the following form:

$$
\begin{gathered}
\log T_{\text {eff }}=3.265-0.3026 \log P_{1}-0.1777 \log M+0.2402 \log L \\
\log Y=-20.26+4.935 \log T_{\text {eff }}-0.2638 \log M+0.3318 \log L \\
\log M=a_{0}+a_{1} \log P_{1}+a_{2} \phi_{31}+a_{3} \log Y \\
\log L=b_{0}+b_{1} \log P_{1}+b_{2} \phi_{31}+b_{4} \log Y
\end{gathered}
$$

Equation (2) is a version of the familiar period/mean-density relation, the socalled "pulsation law," while Eq. (3) describes how the mean location of the firstovertone instability strip changes with the helium parameter, $Y$. We have rendered Eqs. (4) and (5) schematically, since there are a number of different ways in which these fits can be made. The fitting procedure, along with the generalisation of the hydrodynamic models, mentioned above, are discussed in detail elsewhere (Simon and Clement, in preparation; hereafter SCprep).

We note that the parameter list in Eqs. (2) through (5) includes $Y$, but omits the metal abundance, $Z$. This is because, for given mass and luminosity, $Z$ is unimportant in determining either $\phi_{31}$ or $P_{1}$, whereas $Y$ influences the location of the instability strip and thus the mean periods of the models (see also, Simon 1990b). However, it is well known that the calculated blue edges are also influenced by certain (problematical) characteristics of the models, e.g., the boundary conditions and the treatment of convection. We have chosen to subsume all of these uncertainties into the quantity $Y$. Thus, when, below, we infer a large (small) value of $Y$, this could mean either a high (low) abundance of helium in the ionisation zones, or that proper physics and numerics in the code would result in hotter (cooler) blue edges, or both. This question is discussed further by SCprep.

Equations (2) through (5) constitute a set which may be solved for the derived variables $\log M, \log L, \log T_{\text {eff }}$ and $\log Y$ in terms of the observables $\log P_{1}$ and $\phi_{31}$. The following relations result (see SCprep):

$$
\begin{aligned}
& \log M=0.39+0.52 \log P_{1}-0.11 \phi_{31} \\
& \log L=2.41+1.04 \log P_{1}-0.058 \phi_{31},
\end{aligned}
$$

whereupon $\log T_{\text {eff }}$ and $\log Y$ may be calculated from Eqs. (2) and (3).

Because these relations are all linear they may be applied equally well to individual RRc stars or to cluster averages. In Table 2 we show average RRc masses, luminosities, and temperatures for six clusters - five from CJS, plus M68 (Clement, 
Ferance and Simon, in preparation). Derived values of the helium parameter, $Y$, are also given, along with published cluster metallicities. One sees a general increase of mass and luminosity, and a fall in temperature and in $Y$ as $[\mathrm{Fe} / \mathrm{H}]$ gets smaller, with M15 and M68 containing by far the most massive and brightest stars and exhibiting the coolest temperatures. In fact, if the values given in Table 2 are correct, it means that the Oosterhoff effect is due mainly to differences in temperature. That is, the periods in M68, for example, are longer than those in, say, M5 largely because the RRc stars in the former are considerably cooler. In that case, any formulation which attempts to express a period shift at constant temperature (e.g., Sandage 1982) is clearly unjustified.

Table 2

\begin{tabular}{|c|c|c|c|c|c|}
\hline Cluster & {$[\mathrm{Fe} / \mathrm{H}]$} & $Y$ & $\langle M\rangle$ & $\langle\log L\rangle$ & $\left\langle T_{\text {eff }}\right\rangle$ \\
\hline & & & & & \\
NGC 6171 & -0.99 & 0.30 & 0.57 & 1.65 & 7420 \\
M5 & -1.40 & 0.28 & 0.56 & 1.70 & 7300 \\
M3 & -1.66 & 0.28 & 0.63 & 1.71 & 7280 \\
M53 & -2.04 & 0.27 & 0.62 & 1.75 & 7190 \\
M68 & -2.09 & 0.24 & 0.79 & 1.82 & 7070 \\
M15 & -2.17 & 0.24 & 0.80 & 1.83 & 7050 \\
\hline
\end{tabular}

Table 3 shows the results of applying our equations to the individual stars in each cluster. All of the observations are photographic, but those for NGC 6171, M5 and M68 were obtained at a better site and are probably more accurate (CJS). One notices for these clusters (excluding the anomalously long-period star \#76 in M5), a very tight range of intracluster luminosity and temperature, but a large range of mass. Some of the mass scatter may be explained by errors in measuring $\phi_{31}$ [an error of 0.3 in $\phi_{31}$ leads to an error of about 0.03 in the inferred value of $\log M$ from Eq. (6)], but much of it must be real.

To show this, we have made linear fits to the observed $\phi_{31}$ vs. $\log$ (period) relations for NGC 6171, M5 (excluding star \#76) and M68, and obtain slopes of 17.4, 16.2, and 17.7 , respectively, with an average value, $\mathrm{d} \phi_{31} / \mathrm{d} \log P_{1}=17.1$. We note that Eq. (7), obtained strictly from theoretical models, reproduces this value very well, provided that $\log L \sim$ constant. However, it is also clear that Eq. (6) cannot yield the observed slope if $\log M \sim$ constant. In fact, a decrease of $\log M$ with increasing period is required. Using an expression of the form, $\log M=a \log P_{1}+b$, in Eq. (6) and requiring the observed value $\mathrm{d} \phi_{31} / \operatorname{d} \log P_{1}=17$, one easily finds $a=1.3$. Given a typical intracluster period spread (e.g., M5 without star \#76) of $\left|\Delta \log P_{1}\right| \sim 0.1$, we find $|\Delta \log M| \sim 0.13$ or $|\Delta M| \sim 0.15 M_{\odot}$.

It should be pointed out that, while this range is close to what we have actually obtained for M5 (see Table 3), the spread may still be exaggerated. This is due both to errors in measuring $\phi_{31}$ and to the fact that Eq. (6) describes a mean line 
through the theoretical models (with standard deviation 0.03 in $\log M$ ) and must thus be uncertain in any individual case. Nonetheless the argument given above makes it clear that some spread is required - perhaps (guessing on the basis of our error estimates) of the order $0.1 M_{\odot}$. Thus the following picture emerges of the RRc domain on the horizontal branch within a given cluster: narrow in temperature, narrow in luminosity, but with a considerable range in mass, which accounts for the observed range in period. When one turns to the family of clusters, Table 2 shows a substantial spread in intercluster temperature and luminosity as well as in mass.

Finally, we shall briefly mention three independent tests that argue for the validity of Eqs. (6) and (7): 1) Eq. (7) reproduces the observed hierarchy of relative luminosity among a large sample of RRc stars in $\omega$ Centauri; 2) for M68 and M15, Eq. (6) yields mean RRc masses $\left(M \sim 0.80 M_{\odot}\right)$ which agree quite well with the mean masses obtained for the RRd stars in those clusters; and 3) Eq. (7) gives an LMC distance modulus in agreement with that emerging from other methods. These tests shall be described in detail in SCprep.

\section{Questions and comments}

A. N. COX: What is the uncertainty in your $R R c$ masses inferred from $\phi_{31}$ ?

N. R. SIMON: I would not like to state a formal uncertainty. An error of, say, 0.3 in measuring $\phi_{31}$ leads to an error of 0.03 in $\log M$. In addition, the theoretical calculations have been described by mean functions fit to the model sample. The standard deviation of these fits is also about 0.03 in $\log M$. Thus I would say that the uncertainty in individual masses is large - say 10.05 , or even worse in some cases. However, since the uncertainties I mentioned seem to be strictly random, the mean RRc mass derived for a given cluster should be much more accurate.

A. SANDAGE: Am I correct in believing that your RRc masses agree with the RRd masses recently derived by Art Cox (using OPAL opacities), at least in the crude sense that the Oo I stars turn out to be less massive than the Oo II stars, i.e., that the trend of the original Petersen diagram is confirmed?

N. R. SIMON: Yes, I think that is correct.

G. KOVACS: Can you comment on the systematic difference between your luminosities and the evolutionary luminosities for M15?

N. R. SIMON: I have not looked at this carefully yet. I believe that the masses are also involved here, and that the answer depends upon what you take for the $\mathrm{O} / \mathrm{Fe}$ ratio, whether you think the stars are evolved, etc.

G. KOVACS: Combining the theoretical relation for the position of the blue edge with the probably more solid relations involving $\phi_{31}$ might be dangerous because of the large uncertainties involved in the blue-edge position.

N. R. SIMON: That is why we have subsumed all uncertainties in the absolute blue edge location into the helium parameter, $Y$. The results we have obtained won't change much unless the blue (or red) edges turn out to have much stronger (and weirder) dependences on $L$ and $M$ than present calculations show. 
D. WELCH: I don't really know how to say this diplomatically, so I'll just say it. It is unconscionable, in view of the importance of the RR Lyrae luminosities and their $[\mathrm{Fe} / \mathrm{H}]$ dependence, to introduce photographic data into the discussion. RRc stars in the rich fields of globular clusters require modern detectors and reduction techniques. N. R. SIMON: I would be delighted to see such data, provided that the phase coverage is good. A filled-in photographic light curve is, for our purposes, superior to a CCD light curve with holes. This is an important point that $I$ hope the observers will note. J. O. PETERSEN: Concerning photographic vs. modern CCD observations: the old photographic data for $\omega$ Centauri published by Martin give very accurate Fourier parameters because Martin had 350-400 observations of each variable.

J. O. PETERSEN: In your beautiful analysis you have used only one Fourier decomposition parameter, $\phi_{31}$. One should expect that other Fourier parameters also provide valuable information. Have you tried to use two or more parameters simultaneously?

N. R. SIMON: Unfortunately, the present nonlinear pulsation codes are not up to this task. While they seem to model $\phi_{31}$ rather well for the overtone mode (RRc stars), the codes are less successful for $\phi_{21}$. In the case of the fundamental mode (RRab stars), the calculations seem deficient for both $\phi_{21}$ and $\phi_{31}$. With regard to higher-order terms, I don't think the observations are yet precise enough to compare with models.

J. NEMEC: Do you have an explanation for why the longest-period c-type RR Lyrae stars in $\omega$ Cen have periods as long as 0.485 (V47) or 0.534 (V68)? And, why none of the c-type stars with periods longer than 0.35 day are double-mode RR Lyrae stars? N. R. SIMON: No.

E. BELSERENE: In response to Nemec's question about the long periods of RRc stars in $\omega$ Cen: doesn't it help that the RR Lyrae stars in $\omega$ Cen are not ZAHB stars at all, but evolved stars?

J. NEMEC: While it is probably true that the $\omega$ Cen RR Lyrae stars are evolved, isn't it also true that the M15 RR Lyrae stars are also evolved, and many RRd stars are present in M15?

N. R. SIMON: I want to point out that there is a body of opinion (to which I subscribe) holding that the M15 RR Lyraes cannot constitute a largely evolved population, simply because there are so many of them.

\section{References:}

Clement, C. M., Ferance, S. and Simon, N. R., in preparation.

Clement, C. M., Jankulak, M. and Simon, N. R. 1992, Ap. J. 395, 192.

Rogers, F. J. and Iglesias, C. A. 1992, Ap. J. Suppl. 79, 507.

Sandage, A. 1982, Ap. J. 252, 553.

Simon, N. R. 1988, in Pulsation and Mass Loss in Stars, ed. R. Stalio and L. A. Willson (Dordrecht: Reidel), p. 27.

—. 1989, Ap. J. (Letters) 343, L17. 
—. 1990a, M.N.R.A.S. 246, 70.

1990b, Ap. J. 360, 119.

Simon, N. R. and Clement, C. M., in preparation (SCprep).

Table 3 Measured and Derived Parameters for Individual Stars

\begin{tabular}{|c|c|c|c|c|c|}
\hline Star No. & P1 & $\$ 31$ & M & $\log L$ & $\mathrm{Te}$ \\
\hline \multicolumn{6}{|c|}{ NGC 6171} \\
\hline 4 & 0.282 & 3.47 & 0.54 & 1.64 & 7448 \\
\hline 6 & 0.260 & 2.38 & 0.68 & 1.66 & 7442 \\
\hline 15 & 0.289 & 3.55 & 0.54 & 1.64 & 7430 \\
\hline 19 & 0.279 & 3.21 & 0.57 & 1.65 & 7439 \\
\hline 23 & 0.323 & 4.11 & 0.50 & 1.66 & 7356 \\
\hline \multicolumn{6}{|c|}{ M5 } \\
\hline 15 & 0.337 & 3.93 & 0.53 & 1.69 & 7297 \\
\hline 31 & 0.301 & 3.07 & 0.62 & 1.69 & 7345 \\
\hline 35 & 0.308 & 3.31 & 0.59 & 1.69 & 7339 \\
\hline 40 & 0.317 & 3.54 & 0.57 & 1.69 & 7327 \\
\hline 44 & 0.330 & 4.06 & 0.51 & 1.67 & 7331 \\
\hline 55 & 0.329 & 3.53 & 0.58 & 1.70 & 7288 \\
\hline 62 & 0.281 & 2.61 & 0.67 & 1.69 & 7377 \\
\hline 66 & 0.351 & 4.07 & 0.52 & 1.70 & 7266 \\
\hline 73 & 0.340 & 4.16 & 0.50 & 1.68 & 7306 \\
\hline 76 & 0.432 & 4.66 & 0.50 & 1.76 & 7095 \\
\hline 79 & 0.333 & 3.57 & 0.58 & 1.71 & 7278 \\
\hline \multicolumn{6}{|c|}{ M3 } \\
\hline 37 & 0.327 & 2.92 & 0.67 & 1.73 & 7245 \\
\hline 56 & 0.330 & 3.14 & 0.64 & 1.73 & 7253 \\
\hline 75 & 0.314 & 3.02 & 0.64 & 1.71 & 7294 \\
\hline 85 & 0.356 & 3.73 & 0.57 & 1.73 & 7222 \\
\hline 86 & 0.293 & 3.04 & 0.61 & 1.68 & 7372 \\
\hline 107 & 0.309 & 3.07 & 0.63 & 1.70 & 7316 \\
\hline \multicolumn{6}{|c|}{ M53 } \\
\hline 19 & 0.391 & 3.70 & 0.61 & 1.77 & 7120 \\
\hline 21 & 0.339 & 2.80 & 0.70 & 1.76 & 7197 \\
\hline 23 & 0.366 & 3.69 & 0.59 & 1.74 & 7190 \\
\hline 35 & 0.373 & 4.47 & 0.49 & 1.70 & 7235 \\
\hline 36 & 0.373 & 3.22 & 0.67 & 1.78 & 7130 \\
\hline 40 & 0.315 & 2.81 & 0.67 & 1.72 & 7274 \\
\hline 47 & 0.351 & 3.31 & 0.63 & 1.74 & 7203 \\
\hline \multicolumn{6}{|c|}{ M68 } \\
\hline 1 & 0.350 & 2.15 & 0.84 & 1.81 & 7110 \\
\hline 8 & 0.390 & 2.58 & 0.80 & 1.83 & 7031 \\
\hline $13\left(^{*}\right)$ & 0.362 & 1.76 & 0.94 & 1.85 & 7043 \\
\hline 18 & 0.367 & 2.67 & 0.76 & 1.80 & 7101 \\
\hline 24 & 0.376 & 2.67 & 0.77 & 1.81 & 7076 \\
\hline 33 & 0.391 & 2.98 & 0.72 & 1.81 & 7063 \\
\hline 3 & 0.391 & 2.95 & 0.73 & 1.81 & 7060 \\
\hline \multicolumn{6}{|c|}{ M15 } \\
\hline 3 & 0.389 & 3.28 & 0.67 & 1.79 & 7093 \\
\hline 4 & 0.314 & 1.56 & 0.92 & 1.80 & 7174 \\
\hline 5 & 0.384 & $1.7 !$ & 0.98 & 1.88 & 6977 \\
\hline 10 & 0.386 & 2.84 & 0.74 & 1.81 & 7063 \\
\hline 14 & 0.382 & 1.78 & 0.96 & 1.87 & 6989 \\
\hline 17 & 0.429 & 2.40 & 0.88 & 1.89 & 6921 \\
\hline 24 & 0.370 & 2.81 & 0.73 & 1.80 & 7106 \\
\hline 31 & 0.408 & 3.48 & 0.65 & 1.80 & 7059 \\
\hline 35 & 0.384 & 2.89 & 0.73 & 1.81 & 7073 \\
\hline 38 & 0.375 & 2.85 & 0.73 & 1.80 & 7094 \\
\hline 43 & 0.396 & 3.26 & 0.68 & 1.80 & 7072 \\
\hline 54 & 0.400 & 2.05 & 0.92 & 1.88 & 6965 \\
\hline
\end{tabular}

(*) Included according to criterion of CJS, but crowding effects may be significant here (see Clement, Ferance and Simon, in preparation). 